\title{
Supratentorial high-grade gliomas: maximal safe anatomical resection guided by augmented reality high-definition fiber tractography and fluorescein
}

\author{
Sabino Luzzi, MD, PhD, ${ }^{1,2}$ Alice Giotta Lucifero, MD, ${ }^{1}$ Andrea Martinelli, PhD, ${ }^{3}$ \\ Mattia Del Maestro, MD, ${ }^{4}$ Gabriele Savioli, MD, PhD, ${ }^{4,5}$ Anna Simoncelli, MD, ${ }^{6}$ Elvis Lafe, MD, ${ }^{6}$ \\ Lorenzo Preda, MD, PhD, ${ }^{6}$ and Renato Galzio, $\mathrm{MD}^{7}$
}

\begin{abstract}
${ }^{1}$ Neurosurgery Unit, Department of Clinical-Surgical, Diagnostic and Pediatric Sciences, University of Pavia; ${ }^{2}$ Neurosurgery Unit, Department of Surgical Sciences, Fondazione IRCCS Policlinico San Matteo, Pavia; ${ }^{3}$ Department of Science and High Technology, University of Insubria, Como; ${ }^{4} \mathrm{PhD}$ School in Experimental Medicine, Department of Clinical-Surgical, Diagnostic and Pediatric Sciences, University of Pavia; ${ }^{5}$ Emergency Department, IRCCS Policlinico San Matteo, Pavia; and ${ }^{6}$ Department of Diagnostic Radiology and Interventional Radiology and Neuroradiology, University of Pavia, IRCCS Policlinico San Matteo Foundation, Pavia; and ${ }^{7}$ Neurosurgery Unit, Maria Cecilia Hospital, Cotignola, Italy
\end{abstract}

\begin{abstract}
OBJECTIVE The theoretical advantages of augmented reality (AR) with diffusion tensor imaging (DTI)-based highdefinition fiber tractography (HDFT) and sodium fluorescein (F) in high-grade glioma (HGG) surgery have not been investigated in detail. In this study, the authors aimed to evaluate the safety and efficacy profiles of HDFT-F microscopebased AR cytoreductive surgery for newly diagnosed supratentorial HGGs.

METHODS Data of patients with newly diagnosed supratentorial HGGs who underwent surgery using the AR HDFT$F$ technique were reviewed and compared with those of a cohort of patients who underwent conventional white-light surgery assisted by infrared neuronavigation. The safety and efficacy of the techniques were reported based on the postoperative Neurological Assessment in Neuro-Oncology (NANO) scores, extent of resection (EOR), and Kaplan-Meier curves, respectively. The chi-square test was conducted for categorical variables. A p value $<0.05$ was considered statistically significant.
\end{abstract}

RESULTS A total of 54 patients underwent surgery using the AR HDFT-F technique, and 63 underwent conventional white-light surgery assisted by infrared neuronavigation. The mean postoperative NANO scores were $3.8 \pm 2$ and $5.2 \pm$ 4 in the AR HDFT-F group and control group, respectively $(p<0.05)$. The EOR was higher in the AR HDFT-F group ( $p$ $<0.05$ ) than in the control group. With a mean follow-up of 12.2 months, the rate of progression-free survival (PFS) was longer in the study group (log-rank test, $p=0.006$ ) than in the control group. Moreover, the complication rates were $9.2 \%$ and $9.5 \%$ in the study and control groups, respectively.

CONCLUSIONS Overall, AR HDFT-F-assisted surgery is safe and effective in maximizing the EOR and PFS rate for patients with newly diagnosed supratentorial HGGs, and in optimizing patient functional outcomes.

https://thejns.org/doi/abs/10.3171/2021.5.FOCUS21185

KEYWORDS augmented reality; diffusion tensor imaging; fiber tractography; fluorescein; high-grade glioma; neuronavigation

$\mathrm{G}$ LIOMA surgery has two main goals: to maximize the extent of resection (EOR) of the tumor due to the consequent increase in overall survival (OS) and progression-free survival (PFS) rates, and to improve the patient's quality of life (QOL). ${ }^{1-4}$ In doing so, the surgeon needs to have an accurate plan and properly execute the surgical procedures, both based on a deep knowledge of the white matter anatomy of the brain.

ABBREVIATIONS AR = augmented reality; BOLD = blood oxygenation level-dependent; CE = contrast-enhanced; DSC = dynamic susceptibility CE; DTI = diffusion tensor imaging; DWI = diffusion-weighted imaging; EOR = extent of resection; $F$ = sodium fluorescein; fMRI = functional MRI; GTR = gross-total resection; HDFT = high-definition fiber tractography; HGG = high-grade glioma; IDH = isocitrate dehydrogenase; MGMT = $0^{6}$-methylguanine-DNA methyltransferase; NANO = Neurological Assessment in Neuro-Oncology; NTR = near-total resection; OS = overall survival; PFS = progression-free survival; $\mathrm{QOL}=$ quality of life; SLF = superior longitudinal fasciculus; T1W = T1-weighted.

ACCOMPANYING EDITORIAL DOI: 10.3171/2021.5.FOCUS21321.

SUBMITTED March 26, 2021. ACCEPTED May 13, 2021.

INCLUDE WHEN CITING DOI: 10.3171/2021.5.FOCUS21185. 
Diffusion tensor imaging (DTI) high-definition fiber tractography (HDFT) has been proven useful for planning of the brain tumor resection as well as the postoperative assessment of the residual structural anatomical connectivity of the white matter fiber tracts. ${ }^{5-7}$ However, the potential benefits of its intraoperative use have not been extensively studied, with the exception of an experimental level often involving virtual or mixed reality. Contrary to augmented reality (AR), which has recently gained an increasing interest due to its extraordinary feasibility, virtual and mixed realities still have a wide range of limitations related to their implementation in the operative scenario. ${ }^{8-14}$

Sodium fluorescein $(\mathrm{F})$ has been validated as a useful in vivo fluorescent dye in high-grade glioma (HGG) surgery that can increase the EOR and rates of OS and PFS. ${ }^{15,16}$ Nevertheless, it provides no information about the connectivity nor the function of the tumoral tissue, with consequent well-known and nonnegligible risks of iatrogenic damages in the case of unrestricted, yellow-guided tumor resection. ${ }^{17}$

The rationale of the combination of intraoperative HDFT and F dye lies in the possibility of increasing tumor resectability, thus resulting in better patient functional outcomes and QOL. The possibility of full integration in the form of microscope-based AR represents the greatest theoretical advantage for HGG surgery, since the surgeon can simultaneously use both techniques in a single tool.

The primary objective of the present study was to evaluate the safety and efficacy of HDFT-F microscope-based AR during the cytoreductive surgery of newly diagnosed supratentorial HGGs. The reliability and technical aspects of the technique were also analyzed.

\section{Methods}

The demographic, clinical, and surgical data of a consecutive series of patients with newly diagnosed supratentorial HGGs who underwent HDFT-F microscope-based AR-assisted surgery (AR HDFT-F group) (2019-2020) were retrospectively reviewed and compared with those of a cohort of patients who underwent conventional non-AR surgery assisted by infrared neuronavigation, brain mapping, and F (control group) (2018-2020). In the AR HDFT$\mathrm{F}$ group, the surgeries were performed by two neurosurgeons who started to implement the technique when it was available at our institution. Only those patients who underwent concurrent adjuvant chemoradiotherapy were chosen.

\section{Preoperative Workup}

Neurological evaluation was conducted based on the Neurological Assessment in Neuro-Oncology (NANO) scores. ${ }^{18}$ In addition to conventional structural CT and MRI, all patients underwent diffusion-weighted imaging (DWI) and MR spectroscopy. The scheduled AR HDFTF preoperative protocol involved the acquisition of DWI data on a 1.5T MRI scanner (MAGNETOM Symphony, Siemens Healthcare $\mathrm{GmbH}$ ) and evaluation of diffusionweighted 2D echo planar imaging sequences (TR 9000 msec, TE 109 msec, average 4, slices 45, slice thickness $3 \mathrm{~mm}$, FOV $230 \mathrm{~mm}$, matrix $128 \times 128$, spatial resolution $1.8 \mathrm{~mm} \times 1.8 \mathrm{~mm}$ ). The diffusion b-factors, encod- ing directions, and acquisition time were $0-800 \mathrm{sec} / \mathrm{mm}^{2}$, 12 minutes, and 7.6 minutes, respectively. Dynamic susceptibility contrast-enhanced (DSC) MR perfusion and blood oxygenation level-dependent (BOLD) functional MRI (fMRI) were performed on selected patients of both groups based on the tumor location and availability of a trained team. Images were imported on a DICOM imaging workstation (OsiriX DICOM Viewer, Pixmeo), which enabled a detailed calculation of the tumor volume throughout a segmentation of the contrast-enhancing areas on the axial T1-weighted (T1W) MR image. In reporting the tumor location, the central lobe was also considered according to Yaşargil, 19,20 whereas cortical pallial involvement was based on the cytoarchitectonic categorization of the primitive mammalian cortex by Sarnat and Netsky. ${ }^{21}$ The eloquence of the lesions was based on the functional grading system proposed by Lacroix et al. ${ }^{22}$ and adapted from Sawaya et al. ${ }^{23}$ In the study group, the surgical planning involved the transfer of DWI DICOM images on the navigation platform (Brainlab Curve, Brainlab AG) along with the CT, T1W contrast-enhanced (CE) 3D magnetizationprepared rapid gradient echo imaging, 3D T2-weighted images, and FLAIR MR images, which were automatically fused. The Brainlab SmartBrush software was used for DTI-based HDFT. It comprehended a deterministic dual-tensor tracking algorithm with a fiber assignment by continuous tracking, with a minimum fiber length of 100 $\mathrm{mm}$ and fractional anisotropy threshold of 0.1. Moreover, it involved automatic or interactive multiplanar regions of interest selection. The fiber tracking protocol involved the corticospinal tract; arcuate, uncinate, occipitofrontal, superior, and inferior longitudinal fasciculus; optic radiation (Meyer's loop); anterior commissure; and cingulum. The software also allowed a color 3D visualization of the tumor via a semiautomatic multiplanar contouring.

\section{Choice of Approach}

In the AR HDFT-F group, the choice of the approach, including the surgical position, was based on the tumor location; relationships with the neighboring white matter fiber tracts on DTI; dominance of the involved hemisphere; and the patient's age, comorbidities, and neurological status. Apart from the HDFT data, which were not included in the surgical planning, the clinical factors of the patients were the same in the control group. For those HGGs which were either primarily affected or close to the corticospinal tract, cortical-subcortical mapping was employed in both groups; the stimulation technique (direct cortical or transcranial) was decided on a case-by-case basis.

\section{Planning EOR}

In all Lacroix grade I tumors and gyral or lobar grade II tumors of the nondominant hemisphere, the target of surgery was a supratotal removal of the tumor, which was mainly planned on FLAIR and performed via a sulcal-tosulcal resection. However, the EOR was tailored according to the BOLD fMRI and cortical-subcortical mapping. In left-sided grade II and grade III HGGs, a tumorectomy of the contrast-enhancing area on T1W MRI was planned. In deep-seated and subcortical HGGs residing below the sulcal level, a transsulcal parafascicular approach was em- 
ployed. Instead, tumors abutting the cortex were treated using a transgyral approach.

\section{Surgical Workflow in AR HDFT-F Group}

Asleep craniotomy was performed in all patients. After induction of anesthesia, $\mathrm{F}$ was administered at a dose of $5 \mathrm{mg} / \mathrm{kg}$. After CT-based optical tracking registration, an autodetection on the part of the robotic surgical microscope (KINEVO 900, Carl Zeiss) established the integration with the navigation platform. The surgical microscope was equipped with two integrated filters (YELLOW 560 and INFRARED 800, Carl Zeiss). Then, HDFT images were injected into the microscope eyepieces as colored fiber tracts which, as needed, can be overlapped onto the surgical scene at any step of the surgery. Optionally, single tracts can be added or unviewed as virtual modular elements of the AR. It was the same for the tumor, viewable as a 3D solid shape in color. The microscope-based navigation enabled the focus to be used as a probe tip, whereas the microscope eyepiece functioned as a "see-through display" of the AR. Focus adjustments produced variations in the number, types, and amounts of the visualized fiber tracts based on their depth. AR did not limit the surgical workflow. The YELLOW 560 filter enabled the detection of the cortical areas of pathological enhanced fluorescence. The amount of safely resectable fluorescent tissue was determined based on the anatomical relationship with the neighboring fiber tracts and was also confirmed by cortical-subcortical mapping when available. The brainshift correction involved a finite-element model-based elastic fusion.

\section{Surgical Workflow in the Control Group}

Surgeries were performed with two different surgical microscopes (OPMI Neuro-NC4 and KINEVO 900, Carl Zeiss). The decision to stop surgery was based on the surgeon's experience in recognizing tumor boundaries, brain mapping data, and fluorescent enhancement.

\section{Histology, Follow-Up, and Adjuvant Therapy}

The histological types were reported according to the WHO 2016 classification of central nervous system tumors. ${ }^{24}$ The $O^{6}$-methylguanine-DNA methyltransferase (MGMT) methylation status and isocitrate dehydrogenase 1 (IDHI) mutation were also evaluated in some patients according to the availability of the pathology department at the time of surgery. In both groups, the patients underwent an ultra-early postoperative CE MRI within 48 hours, repeated at the first month and every 3 months thereafter. Adjuvant chemoradiotherapy involved the standard Stupp protocol ${ }^{25}$ Bevacizumab was administered to 8 patients in the AR HDFT-F group and 6 patients in the control group.

\section{Imaging Criteria for Residual and Recurrent Tumor, Pseudoprogression/Radionecrosis, and Pseudoresponse}

Images were reviewed for the EOR on the same DICOM imaging workstation by two neurosurgeons and a neuroradiologist who were blinded to the employment of $\mathrm{AR}$ HDFT-F for surgery. On ultra-early postoperative MRI, the criterion for diagnosis of residual tumors was the evidence of variable contrast enhancements, unrestricted on DWI images. During the mid- and long-term imaging follow-up, the diagnosis of local recurrence was supported by 1) CEs on T1W and hyperintensities on T2/FLAIR sequences, 2) increased cerebral blood volume (CBV) and relative CBV (rCBV) on DSC perfusion MRI, and 3) increased choline/ creatine ratio and absolute choline on spectroscopy. The volumes of the remnants and recurrent tumors were calculated through their segmentation. In patients receiving concomitant radiation therapy and temozolomide within the first 3 months after surgery, the occurrence of hyperintensities on T1W CE MRI associated with a low CBV/ rCBV and choline/creatine ratio, with a normal diffusivity, was classified as pseudoprogression/radionecrosis and was managed conservatively.

In patients treated with bevacizumab and in whom a rapid enhancement reduction and vasogenic cerebral edema occurred, the differential diagnosis between tumor progression and pseudoresponse was based on the findings of the perfusion- and diffusion-weighted MRI and MR spectroscopy.

\section{Sample Homogeneity and Outcome Measures}

R (https://www.r-project.org) and Prism 5 (GraphPad Software, Inc.) software were used for statistical analysis. Continuous variables with normal distributions were expressed as mean \pm standard deviation and categorical variables as percentages. To test the homogeneity, the groups were compared using an unpaired t-test for numerical data, the Welch's t-test for continuous variables with different variances, and the chi-square test for categorical variables. For dichotomous variables, the odds ratio was also estimated.

The safety profiles of the AR HDFT-F surgery group and the control group were obtained from patient postoperative neurological assessment and were reported as the average between the postoperative and 3-month NANO score.

The efficacy of each technique was evaluated in terms of the maximal safe EOR and survival curves. The EOR was reported by Chaichana et al. as the percentage ratio between the difference in the preoperative and postoperative tumor volume and preoperative tumor volume. ${ }^{26}$ Gross-total resection (GTR), near-total resection (NTR), and subtotal resection of the EOR were defined as 100\%, $99 \%-98 \%$, and $\leq 97 \%$, respectively. The chi-square test was used to compare the differences between the groups with regard to the pre- and postoperative NANO scores and EOR; $\mathrm{p}$ values $<0.05$ were considered statistically significant in each of the aforementioned tests.

The OS rate was calculated from surgery until the patient's death at the last follow-up, if not censored. The PFS rate was estimated as the time between surgery and the discovery of tumor progression or patient death at the last follow-up, if not censored. Response to the first-line treatment and disease progression was assessed at each follow-up according to the Response Assessment in Neuro-Oncology criteria. ${ }^{27}$ OS and PFS analyses were conducted using the Kaplan-Meier method, and comparisons between the groups were performed using the log-rank test; $p<0.05$ was considered statistically significant. 
TABLE 1. Patient demographic, clinical, and histological data

\begin{tabular}{|c|c|c|c|}
\hline Variable & AR HDFT-F Group & Control Group & $p$ Value* \\
\hline Time frame & Jan 2019-Sep 2020 & Jan 2018-Sep 2020 & \\
\hline No. of patients & 54 & 63 & \\
\hline Mean age, yrs & $54.8 \pm 11$ & $55.4 \pm 11$ & 0.755 \\
\hline \multicolumn{4}{|l|}{ Sex } \\
\hline Male & $30(55.6)$ & $39(61.9)$ & \multirow{2}{*}{0.573} \\
\hline Female & $24(44.4)$ & $24(38.1)$ & \\
\hline Left-sided HGG & $34(63)$ & $39(62)$ & $>0.99$ \\
\hline \multicolumn{4}{|l|}{ Tumor location } \\
\hline Frontal & $12(22.2)$ & $12(19)$ & \multirow{6}{*}{0.932} \\
\hline Central & $10(18.5)$ & $12(19)$ & \\
\hline Parietal & $5(9.3)$ & $7(11.1)$ & \\
\hline Occipital & $3(5.6)$ & $4(6.3)$ & \\
\hline Temporal & $12(22.2)$ & $18(28.6)$ & \\
\hline Limbic & $12(22.2)$ & $10(16)$ & \\
\hline \multicolumn{4}{|l|}{ Cortical pallial involvement } \\
\hline Isocortex (neocortex) & $42(77.8)$ & $53(84.1)$ & \multirow{3}{*}{0.730} \\
\hline Mesocortex & $11(20.4)$ & $9(14.3)$ & \\
\hline Allocortex (paleocortex) & $1(1.8)$ & $1(1.6)$ & \\
\hline \multicolumn{4}{|l|}{ Lacroix functional location grade } \\
\hline I & $29(53.7)$ & $36(57.2)$ & \multirow{3}{*}{0.897} \\
\hline II & $7(13)$ & $7(11.1)$ & \\
\hline III & $18(33.3)$ & $20(31.7)$ & \\
\hline Mean preop tumor volume, $\mathrm{cm}^{3}$ & $12.5 \pm 6$ & $15.1 \pm 7$ & 0.038 \\
\hline \multicolumn{4}{|c|}{ Histological type according to WHO 2016 tumor classification } \\
\hline Anaplastic astrocytoma IDH-mutant 9401/3 & $10(18.5)$ & $5(8)$ & \multirow{6}{*}{0.447} \\
\hline Anaplastic astrocytoma IDH-wildtype $9401 / 3$ & 0 & $2(3.1)$ & \\
\hline Anaplastic astrocytoma NOS 9401/3 & $2(3.7)$ & $3(4.8)$ & \\
\hline Glioblastoma IDH wildtype 9440/3 & $10(18.5)$ & $21(33.3)$ & \\
\hline Glioblastoma IDH mutant 9440/3 & $19(35.2)$ & $16(25.4)$ & \\
\hline Glioblastoma NOS 9440/3 & $13(24.1)$ & $16(25.4)$ & \\
\hline \multicolumn{4}{|l|}{ MGMT status } \\
\hline Methylated & $40(74)$ & $36(57)$ & \multirow{3}{*}{0.172} \\
\hline Unmethylated & $9(16.7)$ & $17(27)$ & \\
\hline NOS & $5(9.3)$ & $10(16)$ & \\
\hline Mean follow-up, mos & $12.2 \pm 5$ & $12.2 \pm 4$ & 0.971 \\
\hline Median OS, days & 382 & 376 & 0.537 \\
\hline
\end{tabular}

NOS = not otherwise specified.

Values represent the number of patients (\%) or mean \pm SD unless indicated otherwise.

* The groups were not statistically different, apart from tumor volume.

\section{Classification of Complications}

Based on the four-grade scale proposed by Landriel Ibañez et al., surgical complications were classified as grade I, non-life-threatening; grade II, requiring surgery; grade III, life-threatening; and grade IV, lethal. ${ }^{28}$

\section{Results}

Overall, 117 patients underwent surgery: 54 in the AR HDFT-F group and 63 in the control group. Patient age ranged from 31 to 78 years, with a mean age of 55 years.
Among the patients, $41 \%$ were female. No differences were observed in the demographic and clinical data aside from the mean tumor volume (Table 1). The mean postoperative NANO scores were $3.8 \pm 2$ and $5.2 \pm 4$ in the AR HDFT-F group and control group, respectively $(\mathrm{p}=0.011)$ (Fig. 1A). The GTR + NTR rate was higher in the AR HDFT-F group than in the control group (OR 3.03, 95\% CI 1.25-8.02; $\mathrm{p}=$ 0.019) (Fig. 1B). Table 2 presents the patient neurological outcomes and resection data. The median follow-up was 12.2 months in both groups.

The OS was similar in both groups (log-rank test, $\mathrm{p}=$ 

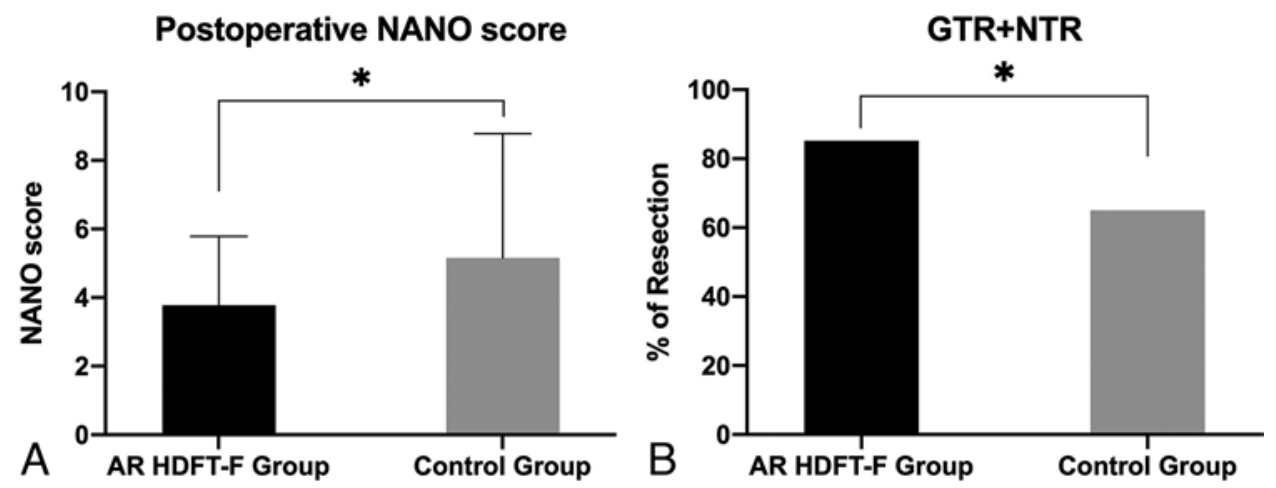

FIG. 1. Bar graphs showing the comparison between the AR HDFT-F group and control group regarding the mean postoperative NANO scores (A) and GTR + NTR rates (B). Error bars represent SD. ${ }^{*} p<0.05$.

0.097), whereas the PFS was longer in the AR HDFT-F group (log-rank test, $p=0.006$ ). Figure 2 presents the OS and PFS curves in both groups.

\section{Complications}

No grade III to IV complications were found in either group, and none of the operative complications were attributable to the AR assistance in the HDFT-F group. Moreover, in the AR HDFT-F group, 5 complications occurred (9.2\%): 2 postoperative hematomas, 1 left caudate-nucleus ischemia in an insular anaplastic astrocytoma, and 2 radionecrosis. In the control group, 2 postoperative hematomas, 1 radionecrosis, 1 thalamic ischemic infarct, 1 hemorrhagic infarct due to occlusion of the vein of Labbé, and 1 pseudoresponse, for a total of 6 complications $(9.5 \%)$, were noted. In both groups, postoperative hematomas were evacuated with no neurological sequelae. The caudate-nucleus ischemia caused mild cognitive and behavioral dysfunction in that patient, which resolved within 1 month. The two patients with radionecrosis were successfully managed with corticosteroids when symptomatic. The thalamic infarct and the occlusion of the vein of Labbé caused hemiplegia in both patients; the patients partially recovered after 6 months of neuromotor rehabilitation.

\section{Illustrative Cases}

\section{Case 1: Primary Motor Cortex Glioblastoma of the Dominant Hemisphere}

A 65 -year-old right-handed female patient experiencing seizures was diagnosed with left primary motor cortex gyral glioblastoma. Preoperative DTI-based HDFT revealed splitting and medialization of the corticospinal tract. The inferior aspect of the lesion also caused an initial caudal displacement of the dorsal component of the superior longitudinal fasciculus (SLF), namely, SLF I, and arcuate fasciculus. Preoperative evaluation of these aspects was important for surgical planning. A transgyral approach was employed with the AR HDFT-F and cortical-subcortical mapping (Fig. 3). Tumor resection was started in the center and progressively carried out toward the peripheric boundaries, achieving the maximal safe EOR while having a constant awareness about the spatial location of the fiber tracts (Fig. 4 and Video 1).

VIDEO 1. AR HDFT and $F$ in the resection of a primary motor cortex glioblastoma of the dominant hemisphere. Copyright Sabino Luzzi. Published with permission. Click here to view.

Postoperative MRI documented a GTR of the tumor, along with the anatomical integrity of the corticospinal tract, SLF I, and arcuate fasciculus (Fig. 3). The patient was discharged without deficits on the 3rd postoperative day. Histology was conclusive for an IDH1-mutated, MGMTmethylated glioblastoma. The treatment was completed by conventional adjuvant therapy, and at the 15-month follow-up, the patient had not experienced recurrence.

\section{Case 2: Insular Anaplastic Astrocytoma}

A 63-year-old male patient with new-onset sleep-related hypermotor epilepsy underwent brain MRI, which

TABLE 2. Patient neurological outcome and resection data

\begin{tabular}{ccccc}
\hline Variable & AR HDFT-F Group & Control Group & OR $(95 \% \mathrm{Cl})$ & $p$ Value \\
\hline Mean NANO score & & & & \\
\hline Preop & $5.1 \pm 2$ & $4.9 \pm 2$ & & 0.663 \\
\hline Postop & $3.8 \pm 2$ & $5.2 \pm 4$ & & \\
\hline EOR & & & & \\
\hline GTR & $44(81.4)$ & $38(60.3)$ & & 0.011 \\
\hline GTR + NTR & $46(85)$ & $41(65)$ & $3.03(1.25-8.02)$ & 0.019 \\
\hline
\end{tabular}

Values represent the number of patients $(\%)$ or mean \pm SD unless indicated otherwise.

${ }^{*}$ Postoperative NANO score and EOR did differ significantly $(p<0.05)$. 

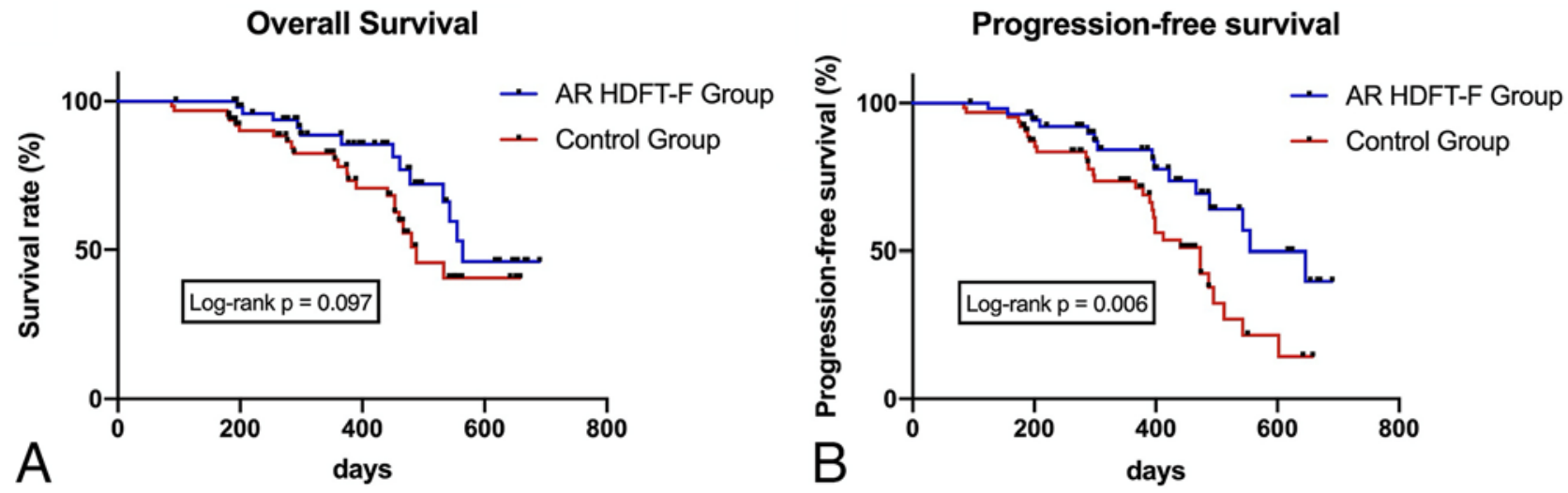

FIG. 2. Kaplan-Meier curves of OS (A) and PFS (B).
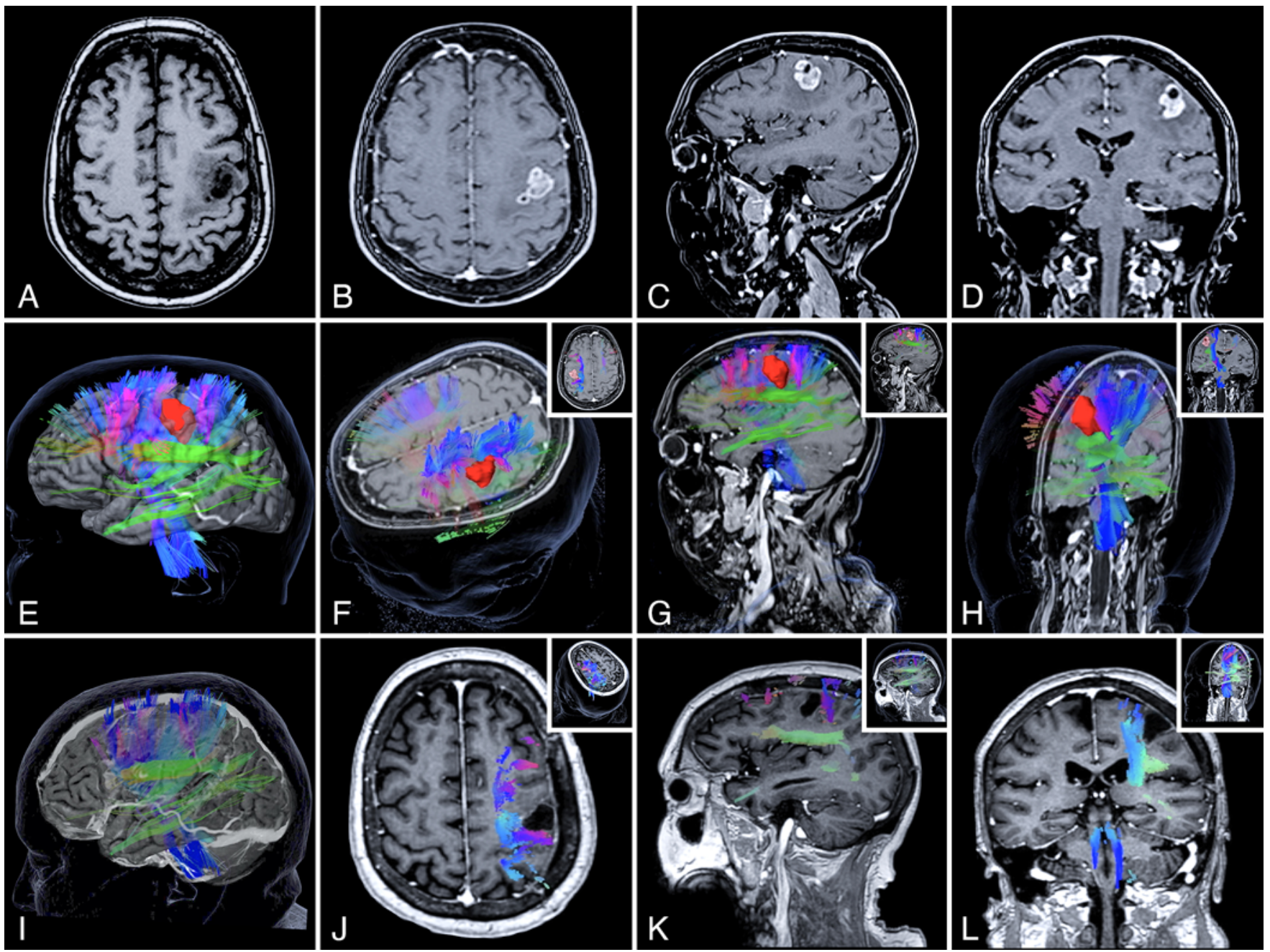

FIG. 3. A-D: Preoperative axial T1W non-CE MR image (A) and axial (B), sagittal (C), and coronal (D) T1W CE MR images showing a left primary motor cortex gyral glioblastoma. E-H: Multiplanar 3D-rendered visualization of the left corticospinal tract and SLF obtained by the T1W CE and HDFT image fusion, used for surgical planning. The tumor was contoured, and the red solid shape corresponded to its volumetric representation. I-L: Postoperative T1W CE and HDFT MR images confirming the GTR of the glioblastoma along with the integrity of the corticospinal tract and SLF. Insets in panels F, G, and $\mathrm{H}$ show the axial, sagittal, and coronal 2D images. Insets in J, K, and L depict the 3D images of the related axial, sagittal, and coronal 2D images, respectively. 


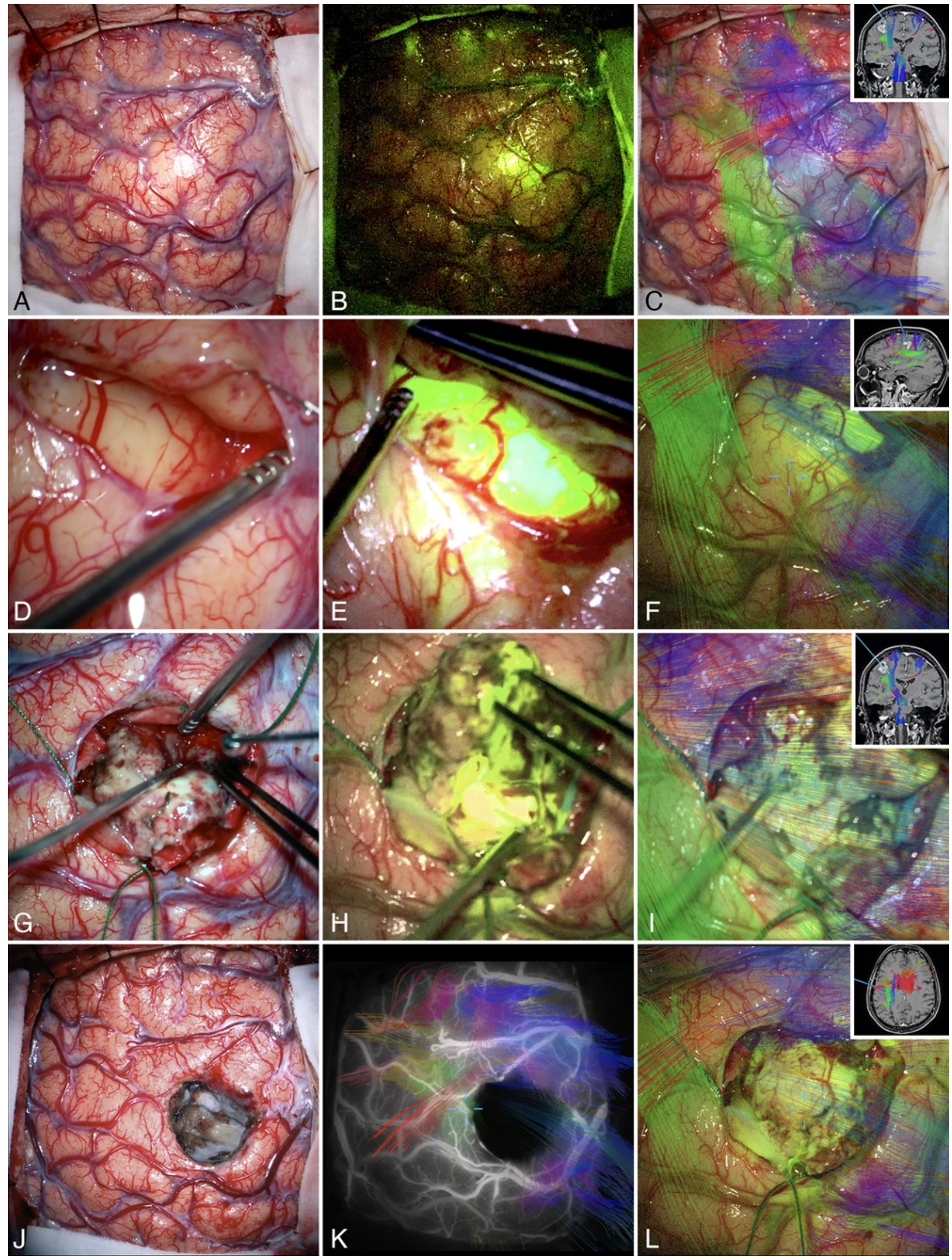

FIG. 4. Intraoperative photographs obtained under white light (A), YELLOW 560 filter (B), and AR HDFT (C) before tumor resection. D-I: The main steps of the surgery that were performed in large part along with the AR HDFT-F technique. FIG. 4. (continued) $\rightarrow$ 
FIG. 4. J-L: The surgical field at the end of the tumor resection obtained under white light (J), combined INFRARED 800 and AR HDFT during indocyanine green videoangiography (K), and AR HDFT-F (L). Insets in panels C, F, I, and L are the screenshots obtained during the microscope focus-based neuronavigation.

revealed a right insular cystic HGG. Preoperative HDFT revealed the anatomical relationships between the tumor, the occipitofrontal fasciculus, and the corticospinal tract (Fig. 5). A pterional transsylvian-transinsular approach was performed with the assistance of AR HDFT-F, transcranial motor evoked potentials, and cortical-subcortical mapping. Intraoperatively, AR HDFT-F was particularly useful during the resection of the deepest part of the tumor, at the interface with the putamen (Fig. 6). Postoperative MRI and HDFT confirmed the GTR, along with the full visualization of the fiber tracts (Fig. 5). The patient was discharged neurologically intact 7 days later, and the histology revealed an IDHI-mutated, MGMT-methylated anaplastic astrocytoma. No recurrence was present at the 21-month follow-up.

\section{Discussion}

The present study aimed to review and critically assess the safety and efficacy of the HDFT-F microscope-based AR surgery in the management of newly diagnosed supratentorial HGGs.

AR HDFT-F assistance was proven to be safe, as no grade III to IV complications occurred and no intraoperative complications directly attributable to the adjunct of AR were observed. The types, grades, and percentages of complications were similar in both the AR HDFT-F and control groups (9.2\% vs 9.5\%). Analogous results about the safety of AR have been reported in other studies. ${ }^{10,29,30}$ The evidence of better postoperative NANO scores in the study group also enabled us to consider the AR HDFT-F assistance among such technical improvements to be useful in achieving better overall functional outcomes and QOL after glioma surgery.

The EOR was significantly higher with the AR HDFT$\mathrm{F}$ assistance, leading to a resection rate of $\geq 98 \%$ in $85 \%$ of the patients. These data are in agreement with the greater intraoperative spatial understanding of the fiber tract course, which ultimately enables surgeons to prevent premature discontinuation of the surgery. The increased EOR was also the logical basis for a longer PFS observed in the study group. OS was almost significant for the study group. Moreover, based on the well-known advantages coming from wider EORs in determining a longer OS in both low-grade gliomas and HGGs, ${ }^{26,31-33}$ it could be concluded that an increased OS can be demonstrated on larger samples of patients. Due to the tremendous progress of the neuroimaging techniques, AR has been recently revitalized and detached from its classic futuristic conception of being a potentially useful and developing tool.

In a recent systematic review, Contreras López et al. reported that brain tumors are the second most frequent intraoperative use of AR. They emphasized that by enabling the surgeon to constantly view the operative field, AR can help in locating fiber tracts and guiding resection, ultimately improving the safety of surgery and patient outcomes. ${ }^{30}$ Ille et al. semiquantitatively delineated the reli- ability and accuracy of virtual dissection of the perisylvian white matter language-related pathways within an AR HDFT environment. They confirmed that AR HDFT is a valuable tool for educational purposes and preoperative planning. ${ }^{34}$ Similar results have been reported by other authors, specifically for AR use in neurooncology. . $^{9,2935-37}$ With regard to AR accuracy, Besharati Tabrizi and Mahvash reported an intraoperative measured projection error of $1.2 \pm 0.54 \mathrm{~mm} .{ }^{35}$ Fick et al. found overlapping results in the accuracy comparisons between AR and conventional infrared neuronavigation systems. ${ }^{8}$ Nevertheless, some biases in the technical error measurement, deriving from head curvatures around tumors, and the incongruence between the 2D AR head images and 3D volumetry should be considered. ${ }^{38}$

\section{Advantages of AR HDFT-F Assistance}

AR HDFT-F increases the surgeon's awareness of the spatial location of the fiber tracts. The integration with the robotic surgical microscope enables the optional use of the heads-up or see-through display. AR HDFT-F allows for a differential visualization of each tract, which can be added or removed from the surgical scene as needed. AR can be used continuously or as needed during the duration of surgery, allowing for the intraoperative visualization of fiber tracts from incision to closure. It is also safe for the patient as it does not involve tracers or dyes. AR HDFT-F can be coupled with indocyanine green videoangiography. The brain-shift correction updates the virtual visualization of the fiber tracts, thus maintaining a high accuracy of neuronavigation. AR HDFT-F can be employed with the use of different microscopes, endoscopes, and ultrasound scanners. It can be coupled with a full-HD exoscopic vision, such as that offered by some new-generation microscopes. However, the present series involved only asleep craniotomies. This is mainly due to the availability of a fully trained team. Our preliminary experience enabled us to collect sufficient information to hypothesize that, during awake craniotomy, AR HDFT-F assistance may offer further advantages in optimizing patient surgical outcomes.

In the near future, the clinical implementation of probabilistic tracking algorithms during surgical planning may allow an intraoperative microscopic mapping of the fiber tracts, further improving the spatial resolution of bundles. A 3D microscopic probabilistic stereotactic map of the main fiber tracts of the human brain has already been reported by Bürgel et al. in 2006. ${ }^{39}$

\section{Technical Limitations of AR HDFT-F Assistance}

The main technical limitation of AR HDFT-F is related to the intrinsic constraint of the DTI ${ }^{40-44}$ DTI provides only anatomical information but no data about the function. For this reason, brain mapping ought to be coupled with the AR HDFT-F technique. Deliberate HDFT- or Fguided resections may potentially lead to an unplanned and detrimental supratotal removal of the lesion, which is 


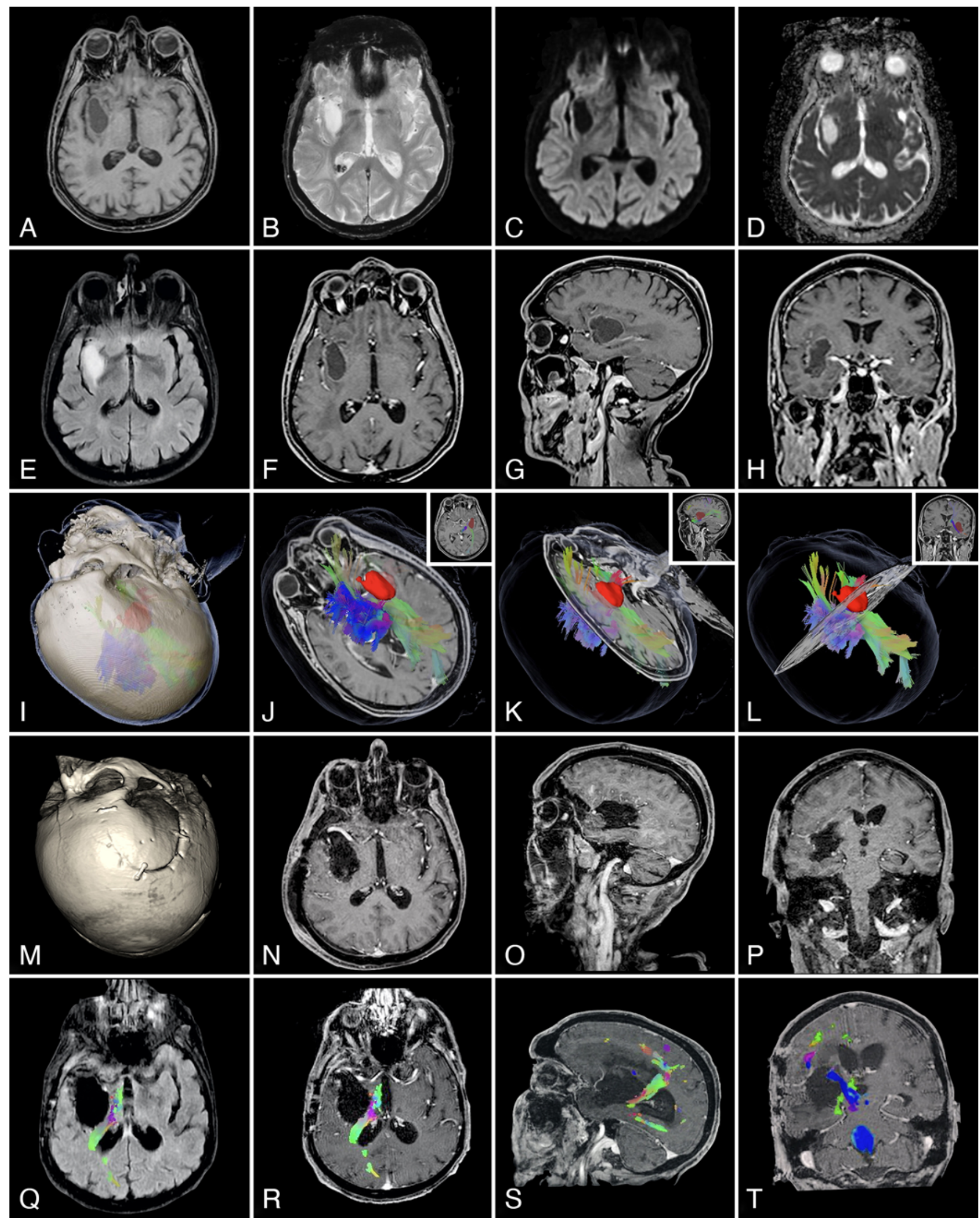

FIG. 5. A-H: Preoperative axial T1W (A), T2*-weighted gradient echo (B), diffusion-weighted (C), apparent diffusion coefficient (D), and FLAIR (E) images and multiplanar axial $(\mathrm{F})$, sagittal $(\mathrm{G})$, and coronal $(\mathrm{H}) \mathrm{T} 1 \mathrm{~W}$-weighted CE MR images. FIG. 5. (continued) $\rightarrow$ 
Luzzi et al.

FIG. 5. I-L: Multiplanar 3D-rendered visualization of the tumor (red solid shape), right corticospinal tract (blue), and occipitofrontal fasciculus (green). M-T: Postoperative multiplanar 3D CT scan (M) and T1W CE MR (N-P), T2 FLAIR+ HDFT (Q), and T1W CE+ HDFT (R-T) images.

considered an overtreatment. Two further limitations have been observed based on our experience, namely, the parallax and the crowding of the fiber tracts. The parallax consists of an optical error common to all the projector-based AR approaches. After the optical tracking registration, the location of virtual images in the operative scene is spatially reliable only if the surgeon's view and the projector have a direct line of sight. ${ }^{1 \text { and }, 38,45}$ The parallax error increases with the depth of the virtual objects or also with the increase in surgeon/projector misalignment. ${ }^{38,45}$ During surgery, this phenomenon is further enhanced by the constant zoom and focus adjustments. Due to the parallax error, Ferrari and Cutolo strongly discouraged the use of AR in tailoring skin incisions, craniotomy, and the du- ral opening for tumor resections. ${ }^{38}$ The crowding of fiber tracts may lead to paradoxical disorientation. An "extravisualization" of fiber tracts may limit or even hide vital structures, making the AR potentially dangerous.

\section{Limitations of the Study}

The limitations of this study include the small number of patients treated with AR HDFT-F assistance. Indeed, our experience ought to be considered as preliminary. Another limitation is that the use of cortical-subcortical mapping intrinsically plays as a potential bias for data interpretation. Since the AR HDFT-F involves a full integration between anatomical information and the functional information about the fiber tracts, we cannot discern whether
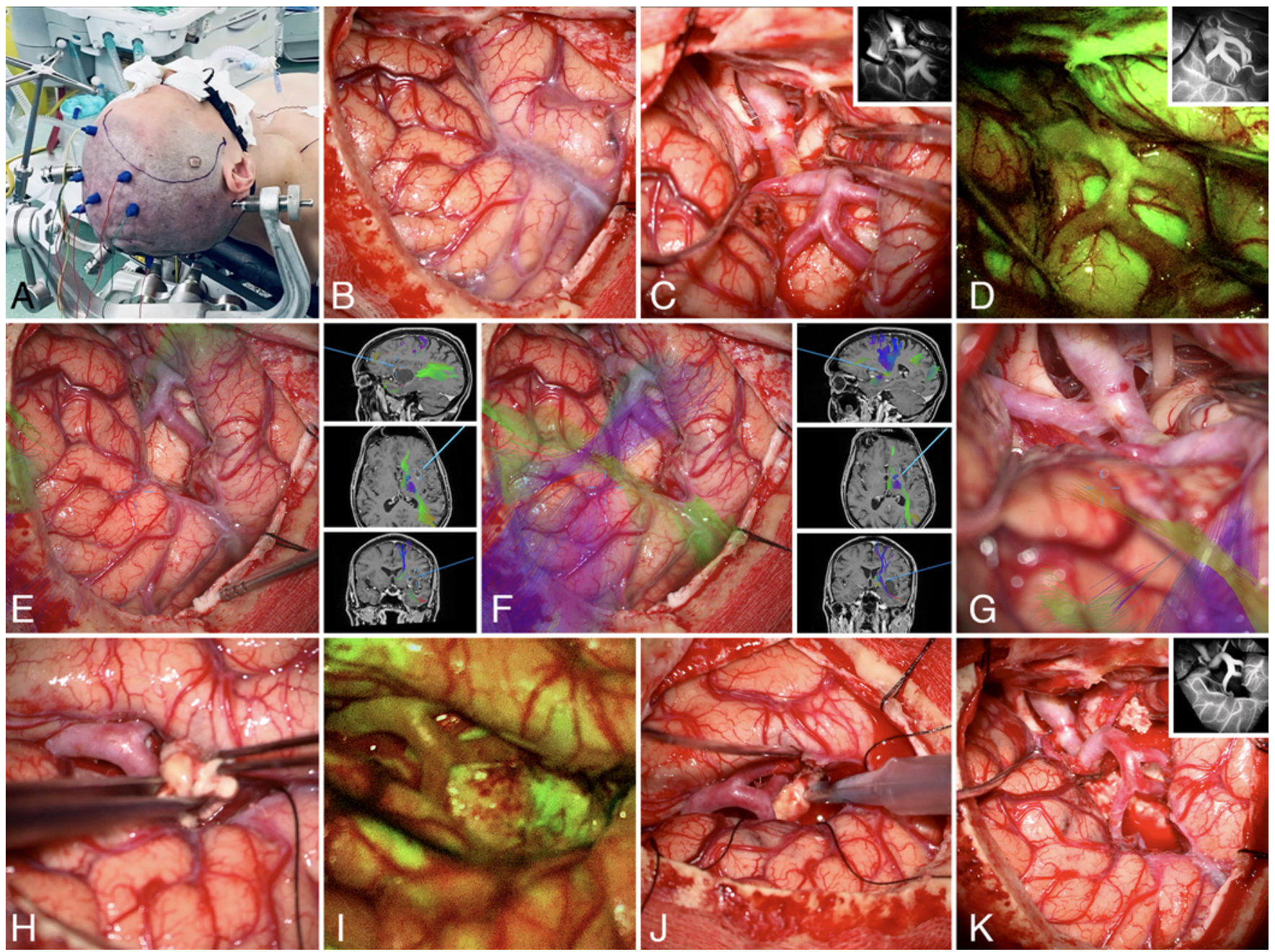

FIG. 6. A-D: The surgical position (A) and main steps of the sylvian fissure splitting under white light (B and $C$ ) and the YELLOW 560 filter (D), the latter revealing the fluorescence uptake by the tumor. In panels $C$ and $D$, the respective frames of the indocyanine green videoangiography are shown as insets. E-G: The variations of the depth of the focus in AR HDFT enabled the visualization and spatial localization of the corticospinal tract and the occipitofrontal fasciculus from the different perspectives of the surgical field. Insets in panels $\mathrm{E}$ and $\mathrm{F}$ show the relative different depths of the focus. H-K: The main steps of the tumor resection performed in AR HDFT-F. In panel K, the respective frame of the indocyanine green videoangiography is shown in the inset. 
the discontinuation of surgery was certainly attributable to the structural anatomical data of the AR HDFT, or partially influenced by the functional information derived from the neuromonitoring. In addition, the different expertise of the surgeons is also a limitation.

\section{Conclusions}

In our preliminary experience, HDFT-F microscopebased AR has been proven to be safe and effective in maximizing the EOR and PFS rate for patients with newly diagnosed supratentorial HGGs. In comparison with the non-AR surgery, it results in a higher postoperative NANO score. The parallax error and crowding of the fiber tracts are shortcomings that still, in part, limit the accuracy and reliability of the technique. Coupling with intraoperative brain mapping techniques should be considered mandatory since the AR HDFT-F technique provides precious information about the structural anatomical connectivity of the fiber tracts but no data about the function.

\section{References}

1. Sanai N, Berger MS. Glioma extent of resection and its impact on patient outcome. Neurosurgery. 2008;62(4):753764, 264-266.

2. Berger MS, Deliganis AV, Dobbins J, Keles GE. The effect of extent of resection on recurrence in patients with low grade cerebral hemisphere gliomas. Cancer. 1994;74(6):1784-1791.

3. Duffau H. Long-term outcomes after supratotal resection of diffuse low-grade gliomas: a consecutive series with 11-year follow-up. Acta Neurochir (Wien). 2016;158(1):51-58.

4. Duffau H. Is supratotal resection of glioblastoma in noneloquent areas possible? World Neurosurg. 2014;82(1-2):e101-e103.

5. Abdullah KG, Lubelski D, Nucifora PG, Brem S. Use of diffusion tensor imaging in glioma resection. Neurosurg Focus. 2013;34(4):E1.

6. Brunberg JA, Chenevert TL, McKeever PE, Ross DA, Junck $\mathrm{LR}$, et al. In vivo MR determination of water diffusion coefficients and diffusion anisotropy: correlation with structural alteration in gliomas of the cerebral hemispheres. AJNR Am J Neuroradiol. 1995;16(2):361-371.

7. Henderson F, Abdullah KG, Verma R, Brem S. Tractography and the connectome in neurosurgical treatment of gliomas: the premise, the progress, and the potential. Neurosurg Focus. 2020;48(2):E6.

8. Fick T, van Doormaal JAM, Hoving EW, Willems PWA, van Doormaal TPC. Current accuracy of augmented reality neuronavigation systems: systematic review and meta-analysis. World Neurosurg. 2021;146:179-188.

9. Kin T, Nakatomi H, Shono N, Nomura S, Saito T, et al. Neurosurgical virtual reality simulation for brain tumor using high-definition computer graphics: a review of the literature. Neurol Med Chir (Tokyo). 2017;57(10):513-520.

10. Meola A, Cutolo F, Carbone M, Cagnazzo F, Ferrari M, Ferrari V. Augmented reality in neurosurgery: a systematic review. Neurosurg Rev. 2017;40(4):537-548.

11. Cutolo F, Badiali G, Ferrari V. Human-PnP: Ergonomic AR interaction paradigm for manual placement of rigid bodies. In: Linte CA, Yaniv Z, Fallovollita, eds. Augmented Environments for Computer-Assisted Interventions. 10th International Workshop, AE-CAI 2015, Held in Conjunction with MICCAI 2015, Munich, Germany, October 9, 2015. Proceedings. Springer; 2015.

12. Karmonik C, Elias SN, Zhang JY, Diaz O, Klucznik RP, et al. Augmented reality with virtual cerebral aneurysms: a feasibility study. World Neurosurg. 2018;119:e617-e622.
13. Jean WC. Mini-pterional craniotomy and extradural clinoidectomy for clinoid meningioma: optimization of exposure using augmented reality template: 2-dimensional operative video. Oper Neurosurg (Hagerstown). 2020;19(6):E610.

14. Edström E, Burström G, Nachabe R, Gerdhem P, Elmi Terander A. A novel augmented-reality-based surgical navigation system for spine surgery in a hybrid operating room: design, workflow, and clinical applications. Oper Neurosurg (Hagerstown). 2020;18(5):496-502.

15. Shinoda J, Yano H, Yoshimura S, Okumura A, Kaku Y, et al. Fluorescence-guided resection of glioblastoma multiforme by using high-dose fluorescein sodium. Technical note. J Neurosurg. 2003;99(3):597-603.

16. Acerbi F, Broggi M, Eoli M, Anghileri E, Cavallo C, et al. Is fluorescein-guided technique able to help in resection of high-grade gliomas? Neurosurg Focus. 2014;36(2):E5.

17. Li Y, Rey-Dios R, Roberts DW, Valdés PA, Cohen-Gadol AA. Intraoperative fluorescence-guided resection of highgrade gliomas: a comparison of the present techniques and evolution of future strategies. World Neurosurg. 2014;82(1-2): 175-185.

18. Nayak L, DeAngelis LM, Brandes AA, Peereboom DM, Galanis E, et al. The Neurologic Assessment in Neuro-Oncology (NANO) scale: a tool to assess neurologic function for integration into the Response Assessment in Neuro-Oncology (RANO) criteria. Neuro Oncol. 2017;19(5):625-635.

19. Yaşargil MG. Microneurosurgery. Vol 4B. Thieme; 1996.

20. Yaşargil MG. Microneurosurgery. Vol 4A. Thieme; 1994.

21. Sarnat HB, Netsky MG. Evolution of the Nervous System. 2nd ed. Oxford University Press; 1981.

22. Lacroix M, Abi-Said D, Fourney DR, Gokaslan ZL, Shi W, et al. A multivariate analysis of 416 patients with glioblastoma multiforme: prognosis, extent of resection, and survival. $J$ Neurosurg. 2001;95(2):190-198.

23. Sawaya R, Hammoud M, Schoppa D, Hess KR, Wu SZ, et al. Neurosurgical outcomes in a modern series of 400 craniotomies for treatment of parenchymal tumors. Neurosurgery. 1998;42(5):1044-1056.

24. Louis DN, Perry A, Reifenberger G, von Deimling A, Figarella-Branger D, et al. The 2016 World Health Organization Classification of Tumors of the Central Nervous System: a summary. Acta Neuropathol. 2016;131(6):803-820.

25. Stupp R, Mason WP, van den Bent MJ, Weller M, Fisher B, et al. Radiotherapy plus concomitant and adjuvant temozolomide for glioblastoma. N Engl J Med. 2005;352(10):987-996.

26. Chaichana KL, Jusue-Torres I, Navarro-Ramirez R, Raza SM, Pascual-Gallego M, et al. Establishing percent resection and residual volume thresholds affecting survival and recurrence for patients with newly diagnosed intracranial glioblastoma. Neuro Oncol. 2014;16(1):113-122.

27. Wen PY, Macdonald DR, Reardon DA, et al. Updated response assessment criteria for high-grade gliomas: response assessment in neuro-oncology working group. J Clin Oncol. 2010;28(11):1963-1972.

28. Landriel Ibañez FA, Hem S, Ajler P, Vecchi E, Ciraolo C, et al. A new classification of complications in neurosurgery. World Neurosurg. 2011;75(5-6):709-715, 604-611.

29. Mikhail M, Mithani K, Ibrahim GM. Presurgical and intraoperative augmented reality in neuro-oncologic surgery: clinical experiences and limitations. World Neurosurg. 2019; 128:268-276.

30. Contreras López WO, Navarro PA, Crispin S. Intraoperative clinical application of augmented reality in neurosurgery: a systematic review. Clin Neurol Neurosurg. 2019;177:6-11.

31. Sanai N, Polley MY, McDermott MW, Parsa AT, Berger MS. An extent of resection threshold for newly diagnosed glioblastomas. J Neurosurg. 2011;115(1):3-8.

32. Zinn PO, Colen RR, Kasper EM, Burkhardt JK. Extent of resection and radiotherapy in GBM: A 1973 to 2007 sur- 
veillance, epidemiology and end results analysis of 21,783 patients. Int J Oncol. 2013;42(3):929-934.

33. Chaichana KL, Zadnik P, Weingart JD, Olivi A, Gallia GL, et al. Multiple resections for patients with glioblastoma: prolonging survival. J Neurosurg. 2013;118(4):812-820.

34. Ille S, Ohlerth AK, Colle D, et al. Augmented reality for the virtual dissection of white matter pathways. Acta Neurochir (Wien). 2020;163(4):895-903.

35. Besharati Tabrizi L, Mahvash M. Augmented reality-guided neurosurgery: accuracy and intraoperative application of an image projection technique. J Neurosurg. 2015;123(1):206-211.

36. Watanabe E, Satoh M, Konno T, Hirai M, Yamaguchi T. The trans-visible navigator: a see-through neuronavigation system using augmented reality. World Neurosurg. 2016;87:399-405.

37. Cho J, Rahimpour S, Cutler A, Goodwin CR, Lad SP, Codd P. Enhancing reality: a systematic review of augmented reality in neuronavigation and education. World Neurosurg. 2020;139:186-195.

38. Ferrari V, Cutolo F. Letter to the Editor: Augmented realityguided neurosurgery. J Neurosurg. 2016;125(1):235-237.

39. Bürgel U, Amunts K, Hoemke L, Mohlberg H, Gilsbach JM, Zilles K. White matter fiber tracts of the human brain: threedimensional mapping at microscopic resolution, topography and intersubject variability. Neuroimage. 2006;29(4):1092-1105.

40. Mori S, van Zijl PC. Fiber tracking: principles and strategies - a technical review. NMR Biomed. 2002;15(7-8):468480 .

41. Duffau H. The dangers of magnetic resonance imaging diffusion tensor tractography in brain surgery. World Neurosurg. 2014;81(1):56-58.

42. Feigl GC, Hiergeist W, Fellner C, Schebesch KM, Doenitz C, et al. Magnetic resonance imaging diffusion tensor tractography: evaluation of anatomic accuracy of different fiber tracking software packages. World Neurosurg. 2014;81(1):144-150.

43. Kinoshita M, Yamada K, Hashimoto N, Kato A, Izumoto S, et al. Fiber-tracking does not accurately estimate size of fiber bundle in pathological condition: initial neurosurgical experience using neuronavigation and subcortical white matter stimulation. Neuroimage. 2005;25(2):424-429.
44. Morita K, Matsuzawa H, Fujii Y, Tanaka R, Kwee IL, Nakada T. Diffusion tensor analysis of peritumoral edema using lambda chart analysis indicative of the heterogeneity of the microstructure within edema. J Neurosurg. 2005;102(2):336-341.

45. Gavaghan K, Oliveira-Santos T, Peterhans M, Reyes M, Kim $\mathrm{H}$, et al. Evaluation of a portable image overlay projector for the visualisation of surgical navigation data: phantom studies. Int J CARS. 2012;7(4):547-556.

\section{Disclosures}

The authors report no conflict of interest concerning the materials or methods used in this study or the findings specified in this paper.

\section{Author Contributions}

Conception and design: Luzzi. Acquisition of data: Luzzi, Giotta Lucifero, Del Maestro, Savioli, Simoncelli, Lafe. Analysis and interpretation of data: Luzzi, Giotta Lucifero, Simoncelli, Lafe. Drafting the article: Luzzi, Giotta Lucifero. Critically revising the article: Luzzi, Giotta Lucifero, Savioli. Reviewed submitted version of manuscript: Luzzi, Giotta Lucifero. Approved the final version of the manuscript on behalf of all authors: Luzzi. Statistical analysis: Luzzi, Martinelli. Administrative/technical/ material support: Luzzi, Giotta Lucifero. Study supervision: Luzzi, Preda, Galzio.

\section{Supplemental Information \\ Video}

Video 1. https://vimeo.com/558037820.

\section{Correspondence}

Sabino Luzzi: University of Pavia, Pavia, Italy. sabino.luzzi@ unipv.it. 